New bri dge ci rcui t-type det ect or to measure preci se resi st ance change of st $r$ ai $n$ gauge at I ow temper at ure and magnet i c fi el d

\begin{tabular}{|l|l|}
\hline 著者 & Ohashi Nasashi, K shi i Nobuya, Tat eno Shot a \\
\hline $\begin{array}{l}\text { j our nal or } \\
\text { publ i cat i on t i t l e }\end{array}$ & Japanese Journal of Appl i ed Physi cs \\
\hline vol une & 55 \\
\hline nunber & 4 \\
\hline page r ange & 046601 \\
\hline year & $2016-04$ 01 \\
\hline URL & ht t p: //hdl . handl e. net /2297/45469 \\
\hline
\end{tabular}




\title{
New bridge-circuit-type detector to measure precise resistance change of strain gauge at low temperature and magnetic field
}

\author{
Masashi Ohashi ${ }^{1,2 *}$, Nobuya Kishii ${ }^{2}$, and Shota Tateno ${ }^{2}$ \\ ${ }^{1}$ Institute of Science and Engineering, Kanazawa University, Kanazawa 920-1192, Japan \\ ${ }^{2}$ Graduate School of Natural Science and Technology, Kanazawa University, Kanazawa 920-1192, Japan
}

\begin{abstract}
We report a new highly accurate and versatile bridge-circuit-type detector that has a simple structure and demonstrates a low degree of error for measurements of thermal expansion and magnetostriction by the strain gauge method. As an example, a commercial physical property measurement system (PPMS) is combined with a compact bridge-circuit box. Thermal expansion and magnetostriction are calculated from the resistance of the bridge and bridge voltage, measured by the operation of a standard PPMS resistivity option. The performance of the new detector is demonstrated by measuring the temperature and magnetic field dependences of the strain to obtain the thermal expansion coefficient and magnetostriction of the single crystals of rare-earth compounds $\mathrm{RAI}_{2}(\mathrm{R}=\mathrm{Dy}, \mathrm{Tb})$.
\end{abstract}

\section{Introduction}

Strain $(\epsilon)$ is defined as the relative change in length, given by $\epsilon=\Delta L / L$. The strain gauge method is widely used for measuring the thermal expansion $\epsilon(T)$ and magnetostriction $\epsilon(B)$ of solid materials. This is because $\epsilon$ can be detected electrically using the relationship $\Delta R / R=K_{S} \epsilon$, where $\Delta R / R$ is the relative change in the electrical resistance of the strain gauge and $K_{S}$ is the gauge factor. Generally, the precision of the measured $\epsilon$ value depends on the specimen conditions and the bridge circuit used to measure the small resistance change of the strain gauge. Previously, we measured thermal expansion and magnetostriction for a wide array of materials such as heavy fermions and superconductors. ${ }^{1-7)}$ To perform these measurements, the sample must be mounted in an apparatus that can control the specimen conditions such as the temperature and magnetic field. On the other hand, it is well known that a commercial physical property measurement system (PPMS; Quantum Design) is a useful apparatus for the generation of extreme conditions and has been used in our previous measurements of physical properties such as electrical resistance and heat capacity for a variety of materials. ${ }^{8-11)}$

*E-mail: ohashi@se.kanazawa-u.ac.jp 
In this paper, we describe a new bridge-circuit-type detector to detect extremely small resistance changes in thermal expansion and magnetostriction measurements using the PPMS.

\section{Design of the bridge-circuit-type detector}

Since $\epsilon$ is proportional to $\Delta R / R$, a small resistance variation $\Delta R$ must be detected to measure a small $\epsilon$ change. The bridge circuit-type detector is one that uses a bridge circuit with a resistance displacement unit $\left(R_{S}\right)$ corresponding to the measurement sensor area on at least one of the four sides. The power supply is located between points $\mathrm{A}$ and $\mathrm{B}$, which face the bridge circuit. It also includes an output voltage measurement unit that detects the voltage $\left(V_{\mathrm{CD}}\right)$ output between points $\mathrm{C}$ and $\mathrm{D}$, which also face the bridge circuit. $V_{\mathrm{CD}}$ produced by the unbalanced bridge circuit is amplified during the measurement owing to the extremely small resistance changes $\Delta R_{\mathrm{S}} / R_{\mathrm{S}}$.

The current PPMS resistivity measurement option adds a configurable resistance bridge board to the Quantum Design 6000 PPMS controller and is a powerful tool for generating electric current to stabilize the current value $i$ and measure the extremely small output voltage $V_{\mathrm{CD}}$. However, in the case of existing bridge-circuit-type detectors, measurement errors may occur for the small relative change $\Delta R_{\mathrm{S}} / R_{\mathrm{S}}$ owing to the internal resistance $(r)$ changes or the changes in the environmental characteristics of the long connecting wire. Furthermore, for thermal expansion and magnetostriction measurements at low temperatures, $R_{\mathrm{S}}$ depends on not only strain $\epsilon$ but also conditions such as temperature and magnetic field of the sample mounted in the cryostat. Since the PPMS applies a constant current to the bridge circuit, power supply voltage changes may occur during the measurement, leading to measurement error for a small relative change $\Delta R_{\mathrm{S}} / R_{\mathrm{S}}$.

Figure 1 shows the new bridge circuit-type detector used in the present study. The detector is quadrilaterally connected to the already known fixed resistors $\left(\mathrm{R}_{1}, \mathrm{R}_{2}\right.$, and $\left.R_{3}\right)$ and to the resistance displacement unit $\left(R_{S}\right)$, where the resistor $\left(R_{1}\right)$ varies with changes in the target physical quantities in the measurement sensor unit used for measuring the physical quantities found in the strain gauge, temperature sensor, and pressure sensor. The power supply unit (E) is located between points A and B of the bridge circuit, and an output pressure measurement unit $\left(\mathrm{V}_{\mathrm{CD}}\right)$ is located between points $\mathrm{C}$ and D. In particular, the proposed detector has a net bridge voltage measurement unit that measures the voltage $V_{\mathrm{AB}}$ that is directly applied to the area between points $\mathrm{A}$ 


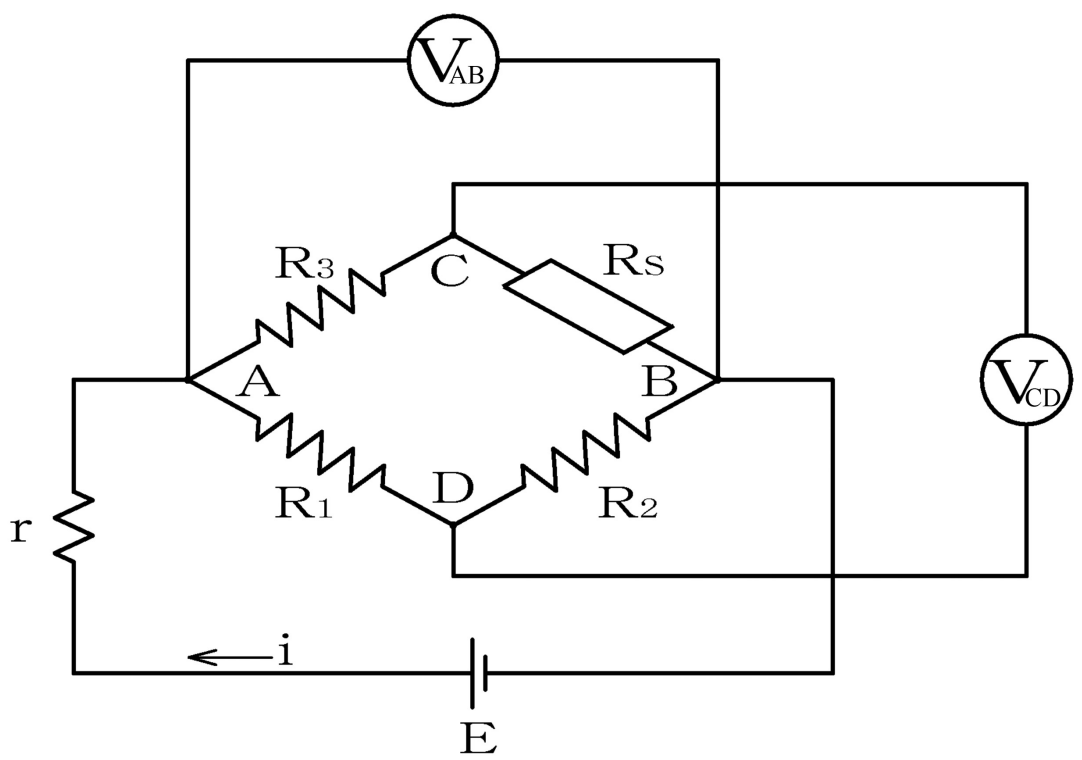

Fig. 1. Composition of the bridge-circuit-type detector.

and $\mathrm{B}$ on the existing device. Because the bridge-circuit-type detector can measure the output voltage $V_{\mathrm{CD}}$ for the area between points $\mathrm{C}$ and $\mathrm{D}$ during $V_{\mathrm{AB}}$ measurement, the obtained values can be used to measure minute resistance changes $\Delta R_{\mathrm{S}} / R_{\mathrm{S}}$ with a high degree of accuracy. Even if resistance changes in the detector, the wire connection cord, and the power supply unit are present owing to the environmental characteristics such as temperature, pressure, and magnetic field within the measurement system, the effect of such environmental changes can be eliminated.

Next, we provide several examples of strain measurement such as tensile strain and compressive strain using a strain gauge on the resistance displacement unit $R_{\mathrm{S}}$.

(1) The initial equilibrium state is set to $R_{1}=R_{2}=R_{3}=R_{\mathrm{S}}$. If the resistance $\left(R_{\mathrm{S}}\right)$ changes when strain $(\epsilon)$ occurs in the strain gauge, the relationship of the output voltage $\left(V_{\mathrm{CD}}\right)$ (the voltage that changes owing to the occurrence of strain $\epsilon$ ) and the net bridge voltage $\left(V_{\mathrm{AB}}\right)$ will be such that the approximate equation $\epsilon=4 / K_{\mathrm{S}} \times V_{\mathrm{CD}} / V_{\mathrm{AB}}$ will hold because of the minuteness of the changes in resistance for $R_{S}$ on account of the gauge factor for the strain gauge being set to $K_{\mathrm{S}}$.

(2) When an active gauge (which is also affected by external factors such as strain, temperature, pressure, and magnetic field) is used for $R_{S}$ and a dummy gauge (which is only affected by external factors such as temperature, pressure, and magnetic field) is used for $R_{3}$ to measure strain, the initial equilibrium state is set to $R_{1}=R_{2}, R_{3}=$ $R_{\mathrm{S}}$. If the resistance $\left(R_{\mathrm{S}}\right)$ changes when strain $(\epsilon)$ occurs in the active gauge, then 
the relationship between the output voltage $\left(V_{\mathrm{CD}}\right)$ (the voltage that changes owing to the occurrence of strain $\epsilon)$ and the net bridge voltage $\left(V_{\mathrm{AB}}\right)$ will be such that the approximate equation $\epsilon=4 / K_{\mathrm{S}} \times V_{\mathrm{CD}} / V_{\mathrm{AB}}$ will hold because of the minuteness of the changes in $R_{S}$ and $R_{3}$ owing to the gauge factor for the strain gauge being set to $K_{\mathrm{S}}$.

(3) In the case where the strain gauge is used to measure the strain (tensile and compressive strains) in $R_{S}$ and $R_{1}$, the initial equilibrium state is set to $R_{1}=R_{2}=$ $R_{3}=R_{\mathrm{S}}$, and if the resistances $R_{\mathrm{S}}$ and $R_{1}$ change when strain $\epsilon$ occurs in the strain gauge, then the relationship of the output voltage $\left(V_{\mathrm{CD}}\right)$ (the voltage that changes owing to the occurrence of strain $\epsilon)$ and the net bridge voltage $\left(V_{\mathrm{AB}}\right)$ will be such that the approximate equation $\epsilon=4 /(1+\nu) K_{\mathrm{S}} \times V_{\mathrm{CD}} / V_{\mathrm{AB}}$ will hold because of the minuteness of the changes in $R_{S}$ and $R_{1}$ as a result of the gauge factor for the strain gauge being set to $K_{\mathrm{S}}$. Here, $\nu$ is a parameter that is determined by the direction of the strain for $\mathrm{R}_{\mathrm{S}}$ and $\mathrm{R}_{1}$.

(4) In cases where the strain gauge is used to measure the strain (tensile and compressive strains) in $R_{\mathrm{S}}$ and $R_{3}$, the initial equilibrium is set to $R_{1}=R_{2}, R_{3}=R_{\mathrm{S}}$, and if the resistances $R_{S}$ and $R_{3}$ change when strain $\epsilon$ occurs in the strain gauge, then the relationship of the output voltage $\left(V_{\mathrm{CD}}\right)$ (the voltage that changes owing to the occurrence of strain $\epsilon$ ) and the net bridge voltage $\left(V_{\mathrm{AB}}\right)$ will be such that the approximate equation $\epsilon=4 /(1+\nu) K_{\mathrm{S}} \times V_{\mathrm{CD}} / V_{\mathrm{AB}}$ will hold because of the minuteness of the changes in $R_{S}$ and $R_{3}$ as a result of the gauge factor for the strain gauge being set to $K_{\mathrm{S}}$. Here, $\nu$ is a parameter that is determined by the direction of the strain for $R_{S}$ and $R_{3}$.

(5) In cases where the strain gauge is used to measure strain (tensile and compressive strains) in $\mathrm{R}_{1}, \mathrm{R}_{2}, \mathrm{R}_{3}$, and $\mathrm{R}_{\mathrm{S}}$, the initial equilibrium is set to $R_{1}=R_{2}=R_{3}=R_{\mathrm{S}}$, and if each of the resistance changes are set to be $\Delta R_{1}, \Delta R_{2}, \Delta R_{3}, \Delta R_{\mathrm{S}}$, and $\Delta R_{1}=\Delta R_{3}=$ $-\Delta R_{2}=-\Delta R_{\mathrm{S}}$ when strain $\epsilon$ occurs in the strain gauge, then the relationship of the output voltage $\left(V_{\mathrm{CD}}\right)$ (the voltage that changes owing to the occurrence of strain $\epsilon$ ) to the net bridge voltage $\left(V_{\mathrm{AB}}\right)$ will be such that the approximate equation $\epsilon=K_{\mathrm{S}} \times V_{\mathrm{CD}} / V_{\mathrm{AB}}$ will hold because of the minuteness of the changes in $R_{S}$ and $R_{1}$ as a result of the gauge factor for the strain gauge being set to $K_{\mathrm{S}}$.

\section{Experimental methods}

As an example of the strain measurement, we demonstrate the bridge-circuit-type detector in which two gauges were used as an active (sample) gauge $R_{\mathrm{S}}$ and a dummy (reference) gauge $\left(R_{3}\right)$. A foil strain gauge, wire strain gauge, or semiconductor strain 


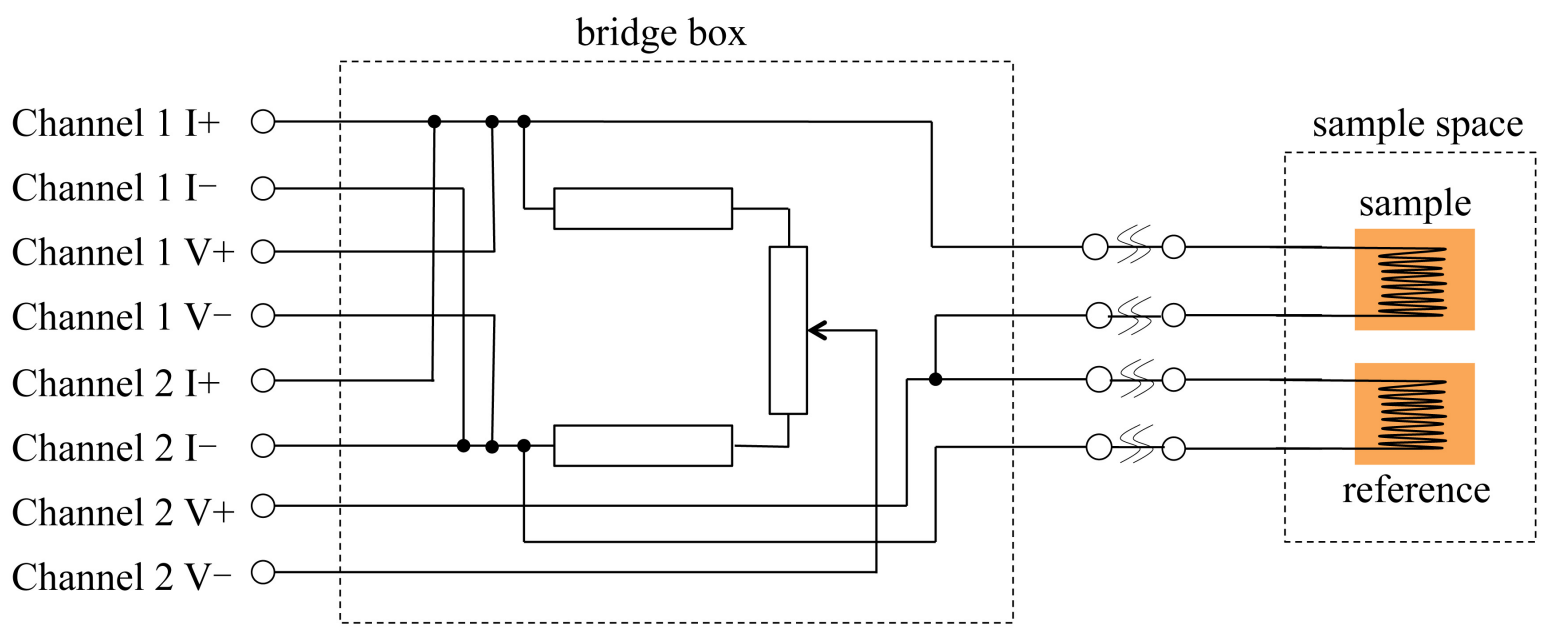

Fig. 2. (Color online) Circuit diagram of the bridge-circuit box.

gauge can all be used as the strain gauge. The gauge factor $K_{\mathrm{S}}$ is a coefficient representing the sensitivity of the strain gauge and has a value of almost 2 for the copper-nickel series and nickel-chromium series alloys used in general-use strain gauges. The strain gauge was glued onto a clean surface of the specimen and cured in accordance with the prescribed directions. The low temperature and the magnetic field were generated using PPMS in the low-temperature laboratory at Kanazawa University. Samples and references are mounted on a sample pack. Strain gauges are connected by electric wires to the contacts of the sample pack. The pack is inserted into the sample chamber in the cryostat.

Figure 2 is a circuit diagram of the bridge-circuit box used in this study. An electrical current flows between I+ and I- on each of channels 1 and 2. An active (sample) and a dummy (reference) gauge are mounted in the sample space of a cryostat, and are connected to the bridge-circuit box by electric wires. A variable resistor is a voltage regulator that is capable of adjusting $V_{\mathrm{CD}}$ to a balanced voltage. $V_{\mathrm{AB}}$ and $V_{\mathrm{CD}}$ correspond to the signals on channels 1 and 2 of a configurable resistance bridge board of the Quantum Design 6000 PPMS controller, respectively.

Figure 3 is a photograph of the bridge-circuit box. Since two independent bridge circuits are present in the box, up to two samples may be mounted on the sample pack and measured simultaneously. The two knobs on the bridge-circuit box are voltage regulators that are capable of adjusting $V_{\mathrm{CD}}$ to a balanced voltage for samples 1 and 2. The bridge-circuit box is connected to the cryostat by a Lemo connecter and to 


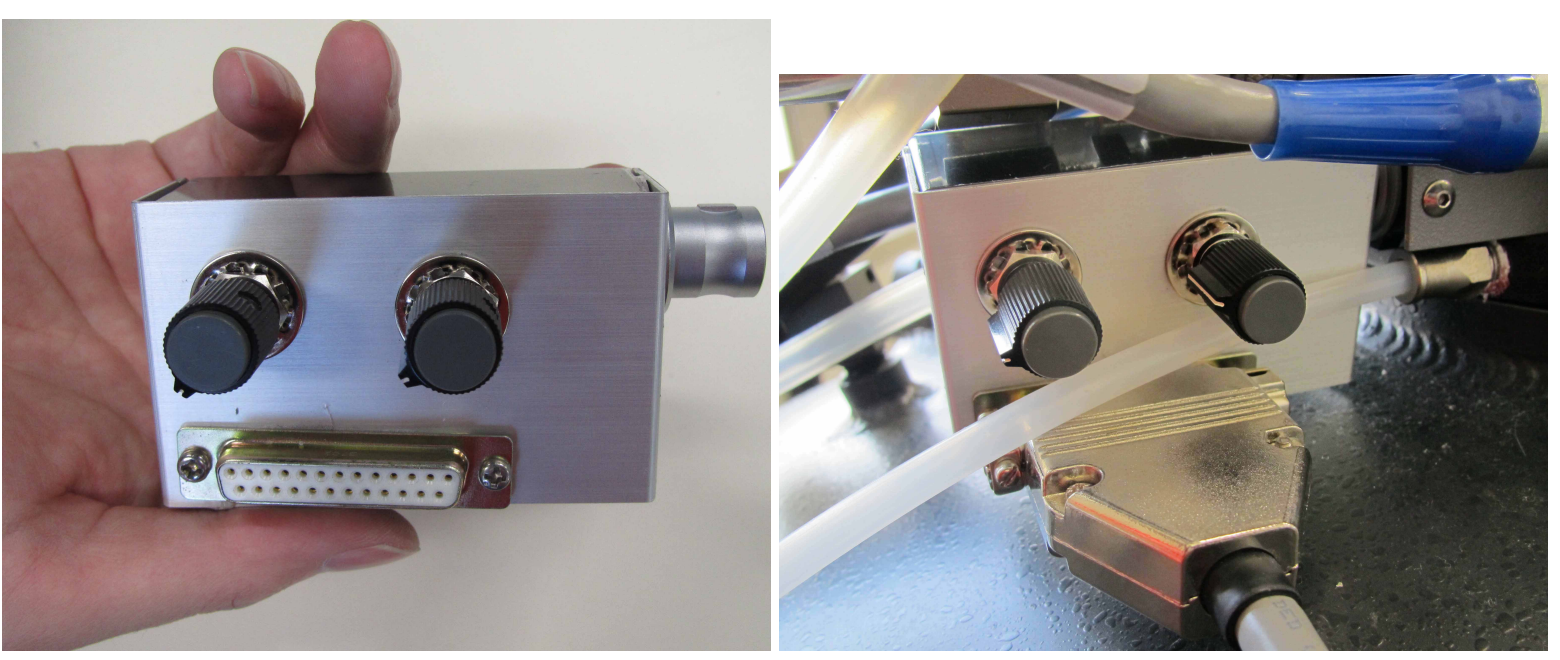

Fig. 3. (Color online) Bridge-circuit box to be attached to the PPMS for strain measurement.

the primary hardware component on the Quantum Design 6000 PPMS controller by a D-sub 25 pin connector. Both $V_{\mathrm{AB}}$ and $V_{\mathrm{CD}}$ can be measured by activating a standard resistivity option on the control $\mathrm{PC}$ of the system. In the case of the bridge-circuit box in Fig. 3, $V_{\mathrm{AB}}$ and $V_{\mathrm{CD}}$ of sample 1 correspond to the signals on channels 1 and 2 of a configurable resistance bridge board of the Quantum Design 6000 PPMS controller, respectively. For sample $2, V_{\mathrm{AB}}$ and $V_{\mathrm{CD}}$ correspond to the signals on channels 3 and 4 , respectively.

\section{Performance}

To demonstrate the use of the new detector in strain measurements, we measured the thermal expansion and magnetostriction of $\mathrm{RAl}_{2}$ (R: rare earth element) single crystals that crystallize in the $\mathrm{MgCu}_{2}$ cubic Laves phase structure ( $F d \overline{3} m$, space group 227). ${ }^{12,13)}$ In most of these compounds, the $\mathrm{R}$ ions are trivalent $(3+)$ and a ferromagnetic ordering caused by local magnetic moments of $\mathrm{R}$ ions has been observed with Curie temperatures $\left(T_{\mathrm{C}}\right)$ of up to $170 \mathrm{~K}^{3,8,9,12)}$ It is expected that some of the structural transition occurs owing to magnetic symmetry breaking below $T_{\mathrm{C}}$.

Single crystals of $\mathrm{RAl}_{2}(\mathrm{R}=\mathrm{Dy}, \mathrm{Tb})$ were grown by the Czochralski pulling method from a melt comprising stoichiometric amounts of the constituent elements in a tetra-arc furnace. ${ }^{14,15)}$ Low-temperature foil strain gauges (Kyowa Electronic Instruments KFL2-120-C1-1) were glued onto a clean surface of the specimen and cured in accordance with the prescribed procedures. The design operating temperature of the strain gauge 
is $4-423 \mathrm{~K}$, the nominal resistance is $120.2 \pm 0.4 \Omega$, and the nominal gauge factor is $K_{\mathrm{S}}=2.01 \pm 0.03$. Copper was used as a reference material. ${ }^{16)}$ An electric current of 1.000 $\mathrm{mA} \pm 2.4 \mu \mathrm{A}$ is produced between points $\mathrm{A}$ and $\mathrm{B}$ of the bridge circuit. To eliminate errors from DC offset voltages, $V_{\mathrm{AB}}$ and $V_{\mathrm{CD}}$ are obtained through $\mathrm{AC}$ measurements using the resistivity option for the PPMS. Taking account of environmental and internal noise sources, the resolution in the most sensitive range of $V_{\mathrm{AB}}$ and $V_{\mathrm{CD}}$ is approximately $20 \mathrm{nV}$.

\subsection{Linear thermal expansion of $\mathrm{TbAl}_{2}$}

Figure 4 shows an example of experimental results for $V_{\mathrm{AB}}$ and $V_{\mathrm{CD}}$ as a function of temperature when the strain of $\mathrm{TbAl}_{2}$ is measured using the bridge-circuit detector and PPMS. Here, the strain gauge is glued along the [111] direction, which is the magnetic easy axis in the ferromagnetic phase. ${ }^{12)}$ Figure $4(\mathrm{a})$ shows that $V_{\mathrm{AB}}$ depends on temperature. This dependence stems from the physical property of the strain gauge used in this study, wherein the resistance decreases with decreasing temperature and shows a minimum at approximately $30 \mathrm{~K}$ in the case of the strain gauge manufactured by Kyowa. ${ }^{17)}$ The results show a difference of approximately $0.3 \%$ in $\Delta V_{\mathrm{AB}} / V_{\mathrm{AB}}$ at approximately $30 \mathrm{~K}$. Such a difference is compensated by measuring $V_{\mathrm{AB}}$ and $V_{\mathrm{CD}}$ at the same time, and the strain is obtained more accurately. On the other hand, as shown in Fig. 4(b), a kink is clearly observed at $T_{\mathrm{C}}=107 \mathrm{~K}$, which corresponds to the ferromagnetic transition temperature. ${ }^{12)}$

The linear thermal expansion of $\mathrm{TbAl}_{2}$ is obtained from the strain $\epsilon=4 / K_{\mathrm{S}} \times$ $V_{\mathrm{CD}} / V_{\mathrm{AB}}$ and the linear thermal expansion of the reference. Figure $5(\mathrm{a})$ shows the temperature dependence of the linear thermal expansion $d L / L$; it is seen that $d L / L$ decreases with decreasing temperature, with the decrease becoming steeper below $T_{\mathrm{C}}$. This indicates that the magnitude of the thermal expansion coefficient below $T_{\mathrm{C}}$ is larger than that above $T_{\mathrm{C}}$. In other words, this material shows a negative magnetostriction, i.e., the length decreasees with the appearance of magnetic ordering. Similar behavior is also observed in other $\mathrm{RAl}_{2}$ ferromagnets. ${ }^{3)}$ For comparison, the results obtained using the existing bridge-circuit and those in the previous report of a $\mathrm{TbAl}_{2}$ polycrystalline sample ${ }^{18)}$ are also plotted. Although the deviation tends to increase with the decrease in temperature, such behavior is a result of various factors such as the temperature dependence of the gauge factor, the sample dependence on the effect of domains in a ferromagnetic sample, the direction of strain in a single crystal, and the difference in 

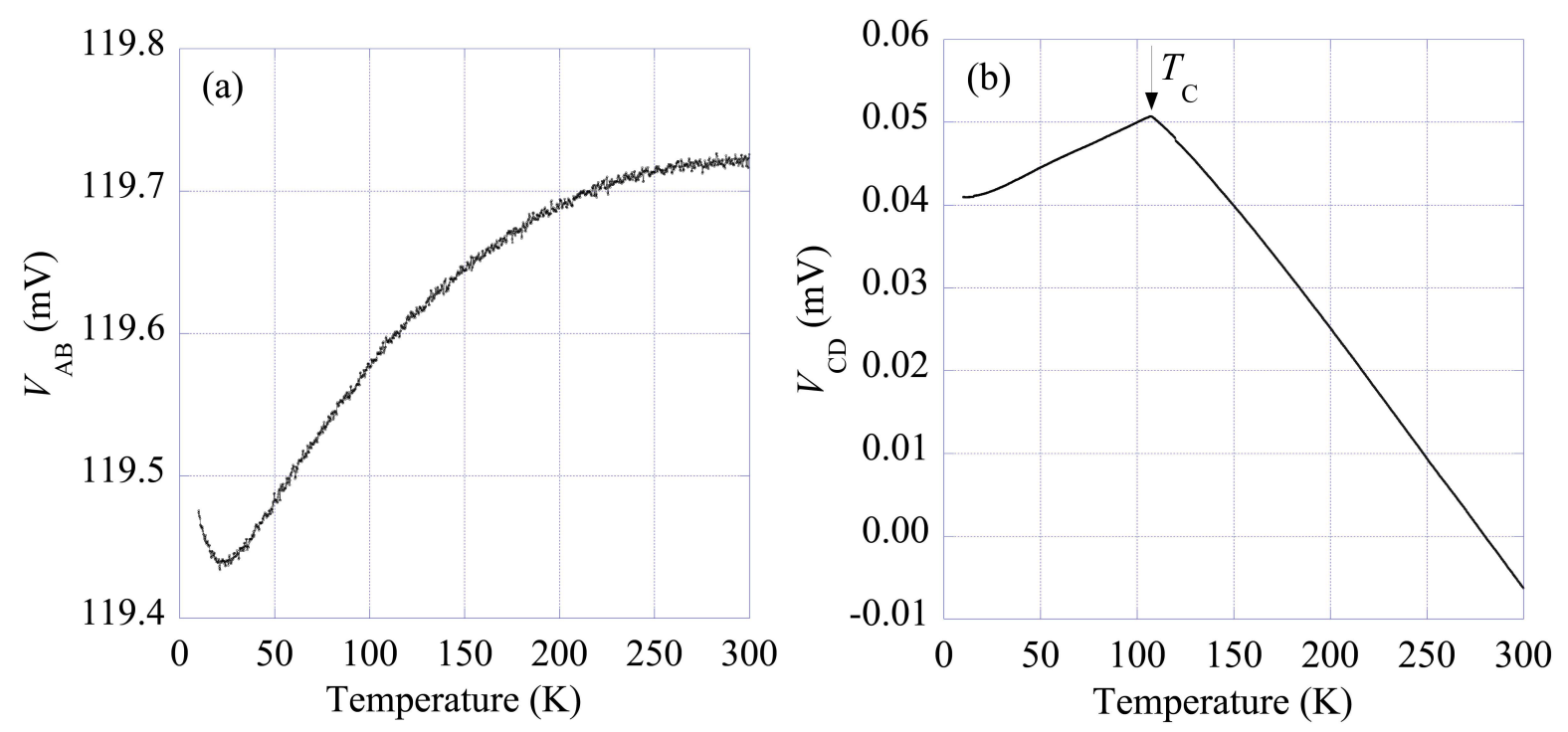

Fig. 4. Example of experimental results of (a) $V_{\mathrm{AB}}$ and (b) $V_{\mathrm{CD}}$ in the strain measurement of $\mathrm{TbAl}_{2}$ along [111] direction as a function of temperature.

the electrical resistances of strain gauges between the sample and the reference.

The linear thermal expansion coefficient $\alpha(T)$ is defined as the derivative of $d L / L$ with respect to temperature, and the experimental results for $\alpha(T)$ are shown in Fig. 5(b). The results obtained using the existing bridge circuit are also plotted for comparison. The jump of $\alpha$ is observed at $T_{\mathrm{C}}$ in both measurements and the behavior of the $\alpha(T)$ curve in the case of using the new detector is qualitatively similar to that when using the existing detector. The deviation between the two results is approximately $\pm 1 \times 10^{-4}$ $\mathrm{K}^{-1}$, which is an acceptable error range in the measurement of thermal expansion by a strain gauge method. ${ }^{1)}$ In the future, we plan to reduce the error by correcting the gauge factor at low temperature. ${ }^{19)}$ Note that the dispersion of the measured data when using the new detector is much smaller than that when using the existing detector. This indicates that there are advantages to measurement using the new detector, at least when measuring a relative amount to detect some phase transition. Since the new detector can be connected to every measurement instrument easily, it is possible to measure the strain precisely by combining the bridge circuit with high-precision instruments such as PPMS. 

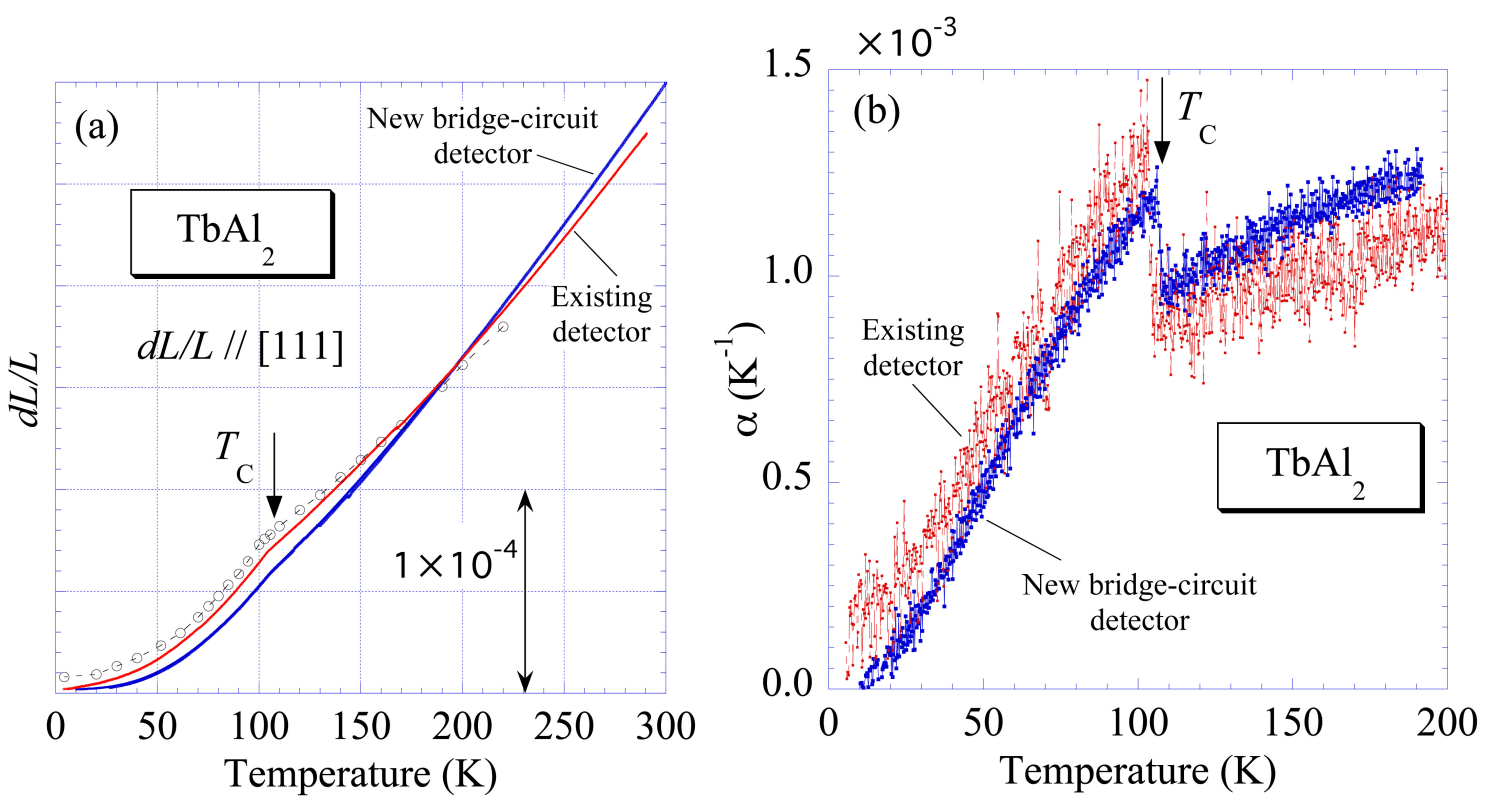

Fig. 5. (Color online) (a) Linear thermal expansion $d L / L$ as a function of temperature along the [111] direction. (b) Thermal expansion coefficient $\alpha$ of $\mathrm{TbAl}_{2}$ as a function of temperature. Blue dots are the results obtained by measurement using the new bridge-circuit detector and red dots the existing detector. Open circles are data taken from the previous report of a polycrystalline sample. ${ }^{18)}$

\subsection{Magnetostriction of $\mathrm{DyAl}_{2}$}

Next, we demonstrate the use of the new detector for measurements of $\mathrm{DyAl}_{2}$ magnetostriction in the low-temperature ferromagnetic phase below $T_{\mathrm{C}}=62 \mathrm{~K}$. The magnetic field sometimes affects the electrical resistance of the strain gauge, and Sakai et al. reported that there is a large difference between longitudinal and transverse magnetoresistance, in which anisotropy $\left[(\Delta R / R)_{\|}-(\Delta R / R)_{\perp}\right]$ at $5 \mathrm{~T}$ is as much as $\left.170 \times 10^{-6} .{ }^{17}\right)$ Therefore, to estimate the anisotropy of the magnetostriction in the new bridge-circuit detector, the effect on the variation in the resistance of the bridge circuit is compensated by measuring $V_{\mathrm{AB}}$ and $V_{\mathrm{CD}}$ at the same time, and the strain is obtained more accurately.

Figure 6 shows the magnetostriction as a function of the magnetic field $B$ for the low temperature ferromagnetic phase of $\mathrm{DyAl}_{2}$ at $10 \mathrm{~K}$. Here, the strain gauge is glued along the [100] axis at room temperature to measure the strain $d L / L$ of one side of a unit cell, and temperature is decreased to $10 \mathrm{~K}$ at zero magnetic field. $d L / L$ is set to zero at $B=0$, and $B$ is applied along the [100] direction, that is, the magnetic easy axis of the $\mathrm{DyAl}_{2}$ ferromagnetic phase. ${ }^{12)}$ Although $d L / L$ shows the strain of one side of a unit cell, the behavior of $d L / L$ is quite different depending on whether $B$ is applied 


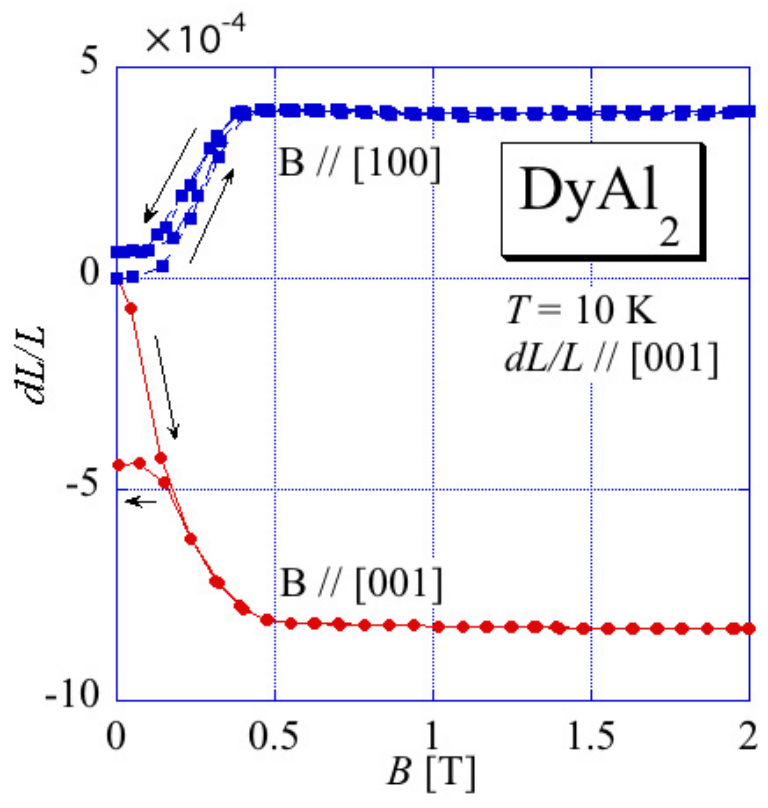

Fig. 6. (Color online) Magnetostriction of $\mathrm{DyAl}_{2}$ as a function of the magnetic field at $10 \mathrm{~K}$.

parallel or perpendicular to the $d L / L$ direction. This behavior is a result of the existence of a large anisotropic exchange interaction between Dy ions, that is, a magnetic order causes low structural symmetry because of a large magnetic anisotropy below $T_{\mathrm{C}}$, while $\mathrm{DyAl}_{2}$ crystallizes in the cubic structure in the room-temperature paramagnetic phase.

When $B$ is perpendicular to the $d L / L$ direction, the magnitude of $d L / L$ increases with increasing $B$, saturating at $4.0 \times 10^{-4}$ above $B=0.3 \mathrm{~T}$. However, when $B$ is parallel to the $d L / L$ direction, the magnitude of $d L / L$ decreases with increasing $B$ and saturates at $-8.0 \times 10^{-4}$ above $B=0.3 \mathrm{~T}$. Considering the crystal symmetry, we assumed that $\mathrm{DyAl}_{2}$ crystallizes in a tetragonal structure in the low-temperature ferromagnetic phase. The $a$-axis then corresponds to $d L / L$ along the direction perpendicular to $B$ in Fig. 6 , and the $c$-axis corresponds to $d L / L$ along the direction parallel to $B$. The axial ratio $c / a$ between the $a$ - and $c$-axes was found to be less than 1 . The X-ray diffractometer can be used to estimate $c / a$ qualitatively.

When the magnetic field is decreased from $B=2 \mathrm{~T}$ to zero, both $d L / L$ values decrease from their saturated values and approach zero at $B<0.3 \mathrm{~T}$. However, $d L / L$ values do not reach their initial zero values. This is because the internal field is still present in the material because of the creation of magnetic domains by the application of the external magnetic field. 


\section{Summary}

We demonstrated precise thermal expansion and magnetostriction measurements using the new bridge-circuit-type detector. This detector is versatile and can be combined with various instruments. For example, strain measurements can be easily conducted at low temperatures down to $2 \mathrm{~K}$ and high magnetic field using PPMS. Furthermore, it is possible to measure strain under various conditions using PPMS combined with several options if the sample and reference are mounted on the sample pack. Compressibility and thermal expansion under high pressure can be measured using the high pressure cell in PPMS. With the use of the PPMS Helium-3 system (P-825) or the dilution refrigerator (P850), strain is easily measured down to 0.4 and $0.05 \mathrm{~K}$. Using the Quantum Design horizontal rotator option, which allows sample rotations around the axis perpendicular to the magnetic field of a longitudinal magnet, we can easily measure the strain as a function of the angle between the direction of the strain gauge and the magnetic field.

\section{Acknowledgments}

This work was performed at the Low Temperature Laboratory, Kanazawa university, and under the Inter-University Cooperative Research Program of the Institute for Materials Research, Tohoku University. This work was supported in part by Grants-in-Aid from the Ministry of Education, Culture, Sports, Science and Technology of Japan, JFE 21st Century Foundation, the Japan Securities Scholarship Foundation, The Kyoto Technoscience Center Foundation, JGC-S Scholarship Foundation, and the Murata Science Foundation. 


\section{References}

1) Y. Uwatoko, I. Umehara, M. Ohashi, T. Nakano, and G. Oomi, in Handbook on the Physics and Chemistry of Rare Earths, (Elsevier, Amsterdam, 2012) Vol. 42, Chap. 252.

2) H. Fujishita, Y Hayashi, M. Saito, H. Unno, H. Kaneko, H. Okamoto, M. Ohashi, Y Kobayashi, and M. Sato, Eur. Phys. J. B 85, 52 (2012).

3) T. Oishi, M. Ohashi, H. Suzuki, and I. Satoh, J. Phys.: Conf. Ser. 200, 082022 (2010).

4) M. Ohashi, G. Oomi, T. Nakano, and Y. Uwatoko, Physica B 378-380, 379 (2006).

5) M. Ohashi, A. Tashiro, G. Oomi, E. Maeda, and X. G. Zheng, Phys. Rev. B 73, 134421 (2006).

6) M. Ohashi, A. Tashiro, G. Oomi, A. Senas, J. R. Fernandez, and J. C. G. Sal, Physica B 329, 1090 (2003).

7) F. Honda, S. Kaji, I. Minamitake, M. Ohashi, G. Oomi, T. Eto, and T. Kagayama, J. Phys.: Condens. Matter 14, 11501 (2002).

8) M. Ohashi, H. Miyagawa, T. Nakano, G. Oomi, V. Sechovsky, I. Satoh, and T. Komatsubara, J. Phys. Soc. Jpn. 83, 024701 (2014).

9) M. Ohashi, H. Miyagawa, T. Nakano, G. Oomi, I. Satoh, and T. Komatsubara, JPS Conf. Proc. 3, 011083 (2014).

10) M. Ohashi, G. Oomi, B. K. Cho, and P. C. Canfield, Phys. Status Solidi B 247, 599 (2010).

11) M. Ohashi and G. Oomi, Jpn. J. Appl. Phys. 48, 070221 (2009).

12) K. H. J. Buschow, Ferromagn. Mater. 1, 297 (1980).

13) H. Miyagawa, M. Ohashi, G. Oomi, I. Satoh, T. Komatsubara, M. Hedo, and Y. Uwatoko, Phys. Rev. B 78, 064403 (2008).

14) M. Ohashi, G. Oomi, and I. Satoh, J. Phys. Soc. Jpn. 76, 114712 (2007).

15) M. Ohashi, G. Oomi, K. Ishida, T. Komatsubara, and I. Satoh, J. Alloys Compd. 408-412, 84 (2006).

16) F. R. Kroeger and C. A. Swenson, J. Appl. Phys. 48, 853 (1977).

17) T. Sakai, T. Kagayama, and G. Oomi, J. Mater. Process. Technol. 85, 224 (1999).

18) F. Pourarian, J. Phys. Chem. Solids 41. 123 (1980).

19) K. Tang, L. Sha, Y. Li, S. Liu, and J. Zhejiang, Univ. Sci. A 15, 323 (2014). 